\title{
Incidence de facteurs alimentaires sur l'obtention de résultats faux positifs lors de la détection des antibiotiques dans le lait par la méthode Delvotest $\mathrm{SP}^{\circledR}$
}

\author{
Jean-Michel Romnee ${ }^{a *}$, Pascale Raskin ${ }^{\mathrm{b}}$, Louis Istasse ${ }^{\mathrm{b}}$, \\ Jean Laloux ${ }^{\mathrm{a}}$, André Guyot ${ }^{\mathrm{a}}$ \\ ${ }^{a}$ Département qualité des productions agricoles, Centre de recherches agronomiques, \\ chaussée de Namur 24, 5030 Gembloux, Belgique \\ ${ }^{\mathrm{b}}$ Service de nutrition, faculté de médecine vétérinaire, université de Liège, \\ Sart Tilman B43, 4000 Liège, Belgique
}

(Reçu le 16 février 1998 ; accepté le 22 octobre 1998)

\begin{abstract}
Incidence of complemented feed on false positive results with Delvotest $\mathrm{SP}^{\circledR}$ for detection of antibiotics in milk. The study described in this paper was performed to show the influence of feed complemented by minerals, oligo-elements and vitamins (CMV) on the results of Delvotest SP ${ }^{\circledast}$. Three trials were developed. In the first trial, a group of 13 dairy cows was divided into two subgroups of six and seven cows, receiving the first one a feed basis without CMV, the second one, the same feed basis added of $100 \mathrm{~g} \mathrm{CMV}$ per day. During the second trial, the dose of CMV was doubled for the last seven cows. During the third trial, all the 8 cows were grazing. The four cows of the test group received $200 \mathrm{~g}$ of CMV per day. The two homogeneous groups of cows (somatic cells, number of lactations) were followed for detection of inhibitors in milk using Delvotest $\mathrm{SP}^{\circledR}$. The test gave positive results with milk from complemented cows during the first and the second trials. All results were negative during the third trial. Feed appears to be a factor that may give false positive results in the determination of antibiotics in milk with Delvotest $\mathrm{SP}^{\circledR}$. (C) Inra/Elsevier, Paris.
\end{abstract}

antibiotic / feed / Delvotest $\mathrm{SP}^{\circledR}$ / false positive

Résumé - On a étudié l'influence de l'alimentation, notamment l'incorporation d'un complément minéral vitaminé (CMV) sur les résultats de la détection des antibiotiques dans le lait par la méthode Delvotest $\mathrm{SP}^{\circledR}$. Trois essais ont été menés. Dans les deux premiers, un groupe de 13 vaches laitières a été divisé en deux lots de six et sept vaches. Le lot témoin (six vaches) recevait l'alimentation de base, le lot traité (sept vaches) l'alimentation de base additionnée de $100 \mathrm{~g} / \mathrm{j}$ de CMV pour le premier

* Correspondance et tirés à part 
essai et de $200 \mathrm{~g} / \mathrm{j}$ de CMV pour le deuxième. Les huit vaches constituant le troisième essai se trouvaient au pâturage. Les quatre vaches du lot traité ont reçu $200 \mathrm{~g} / \mathrm{j}$ de CMV en plus de l'herbe pâturée. Les lots de vaches étaient homogènes du point de vue du nombre de lactations et de la teneur en cellules somatiques. Le Delvotest $\mathrm{SP}^{\mathbb{1 0}}$ a donné des résultats douteux ou positifs uniquement avec le lait des vaches recevant le CMV dans les essais 1 et 2 , mais les résultats étaient négatifs lors de l'essai 3. L'alimentation apparaît done comme un paramètre susceptible de provoquer des résultats faux positifs lors de la détection des antibiotiques dans le lait par la méthode Delvotest SP ${ }^{\circledR}$. (c) Inra/Elsevier, Paris.

antibiotique / alimentation / Delvotest $\mathrm{SP}^{\circledR}$ / faux positif

\section{INTRODUCTION}

Les antibiotiques sont couramment utilisés pour la gestion de l'état sanitaire des troupeaux laitiers. Cette utilisation, incontournable pour des raisons économiques, doit être limitée pour des raisons d'hygiène publique et pour des raisons technologiques. En effet, des résidus peuvent passer dans le lait où ils peuvent présenter un danger pour le consommateur et pour le transformateur [1]. Aussi, la législation en la matière est très sévère [2], favorisant ainsi le développement de méthodes de détection de plus en plus sensibles.

La législation belge préconise un schéma de détermination des substances inhibitrices dans le lait en deux étapes, une première étape de tri basé sur un test microbiologique, l'inhibition de Bacillus stearothermophilus var. calidolactis et une étape de confirmation, relative à la présence de sulfamides et de $\beta$-lactamines sensibles à la pénicillinase [3].

Le lait cru contient des composants naturels qui inhibent la croissance bactérienne et peuvent donner des résultats positifs aux tests microbiologiques. On les dénature par chauffage des échantillons à $80^{\circ} \mathrm{C}$ pendant $10 \mathrm{~min}$. Certaines observations laissent penser qu'un nombre élevé de cellules (supérieur à 500000 cellules $\cdot \mathrm{mL}^{-1}$ ) serait à l'origine de réponses positives au test de sélection (données non publiées), mais ces réponses ne paraissent pas avoir été confirmées. L'identification d'un inhibiteur inconnu est difficile et on ignore le plus souvent à quel niveau et selon quel mode d'action il agit. On sait cependant que les systèmes enzymatiques du Tait jouent un rôle important [4].

Durant ces deux dernières années, des résultats positifs ont été obtenus lors de l'épreuve de tri au Delvotest $\mathrm{SP}^{\circledR}{ }^{\circledR}$ (GistBrocades, Pays-Bas) pour la recherche d'antibiotiques dans le lait de plusieurs exploitations laitières du Sud de la Belgique, alors que l'organisation de ces exploitations excluait pratiquement toute livraison de lait contaminé par des antibiotiques : les producteurs marquaient systématiquement les vaches traitées et éliminaient le lait suspect. Un contrôle individuel du lait destiné à la collecte a montré que 30 à $50 \%$ des vaches produisaient un lait positif au Delvotest $\mathrm{SP}^{\circledR}$. Ces résultats apparaissaient généralement après un changement du régime alimentaire, notamment à l'occasion de l'apport d'un complément minéral.

Notre étude vise à infirmer ou à confirmer une relation entre l'ajout d'un complément minéral et une réponse positive au Delvotest SP ${ }^{\circledR}$.

\section{MATÉRIEL ET MÉTHODES}

Trois essais ont été menés en collaboration entre la station laitière du Centre de recherches agronomiques de Gembloux et le Service de nutrition de la faculté de médecine vétérinaire de Liège disposant d'une ferme expérimentale. 
Les deux premiers essais ont mobilisé 13 vaches laitières réparties en deux lots de sept et six vaches homogènes quant au taux cellulaire du lait, au nombre de lactations et au volume de production.

Lors du premier essai, sept vaches (lot traité) ont reçu lors de la traite, un complément minéral vitaminé (CMV) journalier de $100 \mathrm{~g}$, les six autres vaches (lot témoin) n'ont pas reçu ce complément. Le reste de l'alimentation était constitué pour les deux lots d'une ration à base d'ensilage de maïs ( $7 \mathrm{~kg})$, d'ensilage d'herbe $(7 \mathrm{~kg})$, de pulpes séchées, luzerne et soja $(0,5 \mathrm{~kg})$. La composition du CMV est indiquée dans le tableau 1 .

Lors du deuxième essai, les sept vaches du lot traité (identiques à celles du premier essai) ont reçu $200 \mathrm{~g} \cdot \mathrm{j}^{-1}$ du même CMV. Le complément était distribué lors de la traite, en même temps qu'un peu d'ensilage de maïs afin de rendre le produit plus appétissant. Ces deux essais se sont déroulés en mars, en période de stabulation. Entre ces deux essais, les vaches ont reçu l'alimentation de base. Les résultats obtenus par le Delvotest $\mathrm{SP}^{\circledR}$ étaient négatifs pour toutes les vaches au début de l'essai 2.

Le troisième essai a concerné seulement huit vaches réparties en deux groupes de quatre. Il a été mené durant le mois de juin, en période de pâturage. Quatre vaches ont reçu l'alimentation de base, soit de l'herbe en pâture et $1 \mathrm{~kg}$ d'orge. Ce régime était additionné de $200 \mathrm{~g} \cdot \mathrm{j}^{-1}$ du même CMV. Les quatre autres vaches recevaient exclusivement l'alimentation de base.

Durant les trois essais, deux échantillons de lait de chaque animal ont été prélevés à chaque traite, matin et soir, pendant cinq jours. Sur le premier échantillon, nous avons effectué la détection des inhibiteurs en utilisant le Delvotest SP ${ }^{\circledR}$. La lecture des tests intervenait lorsque la couleur mauve de la gélose du test correspondant à un lait exempt d'inhibiteurs virait au jaune sous l'effet de la baisse de $\mathrm{pH}$. Le deuxième échantillon était envoyé à un laboratoire de contrôle laitier pour la détermination du nombre de cellules somatiques. Le Delvotest $\mathrm{SP}^{\circledR}$ a été effectué sur lait cru et sur lait chauffé $10 \mathrm{~min}$ à $80^{\circ} \mathrm{C}$. Les échantillons positifs ont subi un test supplémentaire en présence de pénicillinase : $15 \mu \mathrm{L}$ de pénicillinase (Bacto ${ }^{\circledR}$ Difco) à une concentration de $40000 \mathrm{UI} \cdot \mathrm{mL}^{-1}$ ont été ajoutés aux $100 \mu \mathrm{L}$ de lait utilisés pour le Delvotest $\mathrm{SP}^{\circledR}$. Les incubations pour les différentes séries de tests ont été conduites à $63{ }^{\circ} \mathrm{C} \pm 1{ }^{\circ} \mathrm{C}$ en étuve ventilée.

Deux échantillons de lait (vache $\mathrm{n}^{\circ} 40$, essai 1 , jour $3 \mathrm{~S}$ et jour $4 \mathrm{M}$ ) donnant une réponse positive au Delvotest $\mathrm{SP}^{\circledR}$ ont été soumis au Charm HVS-8100, appareil permettant l'identification de cinq groupes d'antibiotiques ( $\beta$-lactamines, tétra-

Tableau I. Composition du complément minéral vitaminé.

Table I. Composition of complement (minerals and vitamins).

\begin{tabular}{lrlr}
\hline Oligo-éléments $\left(\mathrm{mg}^{\mathrm{k}} \mathrm{kg}^{-1}\right.$ ) & & & \\
Fer (sulfate) & 7000 & Zinc (oxyde) & 5000 \\
$\mathrm{Cu}$ (sulfate) & 1000 & Iode (iodate de Ca) & 100 \\
Manganèse (oxyde) & 5000 & Sélénium (sélénite de Na) & 10 \\
Cobalt (sulfate) & 50 & & \\
Minéraux (\%) & & & 5 \\
Calcium & 18 & Sodium & 4 \\
Phosphore & 9 & Magnésium & \\
Vitamines (/kg) & & & $20 \mathrm{mg}$ \\
A & $1000000 \mathrm{UI}$ & $\mathrm{B}_{1}$ & $75 \mathrm{mg}$ \\
D & $200000 \mathrm{UI}$ & $\mathrm{B}_{2}$ & $100 \mathrm{mg}$ \\
E & $1000 \mathrm{mg}$ & $\mathrm{B}_{3}$ & $15 \mathrm{mg}$ \\
K & $3 \mathrm{mg}$ & $\mathrm{B}_{6}$ & $0,2 \mathrm{mg}$ \\
PP (niacine) & $155 \mathrm{mg}$ & $\mathrm{B}_{12}$ & \\
Chlorure de choline & $10000 \mathrm{mg}$ & &
\end{tabular}


cyclines, sulfamides, macrolides et aminoglycosides) et une évaluation de la concentration du groupe présent par rapport à la limite maximale résiduelle.

Avant chaque période d'essai, on a vérifié que le lait des vaches suivies donnait une réponse négative au Delvotest SP ${ }^{\circledR}$. L'absence d'agent inhibiteur dans le complément minéral vitaminé a aussi été vérifié après mise en suspension ( $1 \%$ et $0,1 \%$ ) dans du lait.

\section{RÉSULTATS}

\subsection{Essais 1 et 2}

\subsubsection{Caractéristiques des laits}

Le niveau du lait en cellules somatiques de chaque vache, le numéro de lactation et la production journalière de lait produit figurent au tableau $I I$.

Tableau II. Caractéristiques des vaches suivies lors des essais 1 et 2 (cellules, numéro de lactation, production).

Table II. Characteristics of cows for trials 1 and 2 (cells count, number of lactation and production).

\begin{tabular}{ccrr}
\hline Vache & Lactation & $\begin{array}{r}\text { Production } \\
\left(\mathrm{kg} \cdot \mathrm{j}^{-1}\right)\end{array}$ & $\begin{array}{r}\text { Cellules } \\
(\text { par } \mathrm{mL})\end{array}$ \\
\hline $\begin{array}{c}\text { Lot témoin } \\
18\end{array}$ & 5 & 22,3 & 347000 \\
24 & 4 & 26,7 & 56000 \\
26 & 6 & 23,2 & 68000 \\
41 & 1 & 21,7 & 29000 \\
42 & 1 & 22,6 & 32000 \\
45 & 2 & 13,0 & \\
& & & \\
Lot traité & & & \\
4 & 4 & 21,3 & 23000 \\
7 & 8 & 8,0 & 297000 \\
11 & 3 & 24,4 & 8000 \\
13 & 4 & 21,7 & 336000 \\
23 & 2 & 20,3 & 100000 \\
28 & 6 & 26,8 & 43000 \\
40 & 1 & 22,4 & 84000 \\
\hline
\end{tabular}

\subsubsection{Delvotest $S P^{\circledR}$}

Les résultats obtenus sur lait cru (lot traité) par le Delvotest SP ${ }^{\text {ir }}$ lors des deux premiers essais figurent au tableau III. Les résultats obtenus après chauffage du lait ne différaient pas des résultats sur lait cru. Toutes les vaches du lot témoin ont donné, pour le premier essai, un lait dont la réponse était négative.

On remarque des résultats positifs ou douteux pour quatre des sept vaches recevant le CMV. On n'observe pas de relations entre les résultats positifs et le taux de cellules somatiques (tableau II) qui, il est vrai, était inférieur à 400000 cellules. $\mathrm{mL}^{-1}$ pour le lait de toutes les vaches.

En ce qui concerne la vache $n^{\circ} 40$, la réponse était nettement positive. Cette vache a vêlé pour la première fois deux mois auparavant, de sorte que la période colostrale ne peut pas être incriminée. L'arrêt de la complémentation a entraîné la disparition de tous les résultats positifs $4 \mathrm{j}$ plus tard, soit le premier jour de prélèvement de l'essai 2.

Lors du second essai, six vaches sur sept du lot traité donnaient un lait à réponse douteuse au Delvotest $\mathrm{SP}^{\circledR}$ tandis que cinq vaches sur six du lot témoin donnaient un lait à réponse toujours négative. Une vache du lot témoin produisait du lait en fin de l'essai dont la réponse au Delvotest $\mathrm{SP}^{\circledR}$ était douteuse.

L'essai 2 confirme donc l'influence de la complémentation minérale vitaminée. Pour trois des quatre vaches dont le lait avait répondu positivement lors du premier essai, le résultat était semblable mais avait tendance à apparaître plus tôt (dès le lendemain du début de l'essai 2 au lieu de 3 j après le début de l'essai 1). Trois autres vaches recevant le CMV ont produit du lait donnant une réponse positive au Delvotest $\mathrm{SP}^{\circledR}$. Par contre, une des vaches ayant répondu positivement lors du premier essai a donné du lait à réponse négative durant la période du second essai. 
Tableau III. Essais 1 et 2 : Détection des inhibiteurs par le Delvotest $\mathrm{SP}^{\circledast}$ sur le lot recevant le complément minéral vitaminé.

Table III. Trials 1 and 2 : Detection of inhibitors by Delvotest $\mathrm{SP}^{\circledR}$ (cows receiving complemented feed).

\begin{tabular}{|c|c|c|c|c|c|c|c|c|c|c|c|c|}
\hline \multirow{4}{*}{$\begin{array}{l}\text { Vache } \\
n^{\circ}\end{array}$} & \multicolumn{12}{|c|}{ Nombre de jours après le début de l'essai } \\
\hline & \multicolumn{5}{|c|}{ Essai 1} & \multicolumn{7}{|c|}{ Essai 2} \\
\hline & 0 à 2 & 3 & 3 & 4 & 4 & 0 & 1 & 1 & 2 & 2 & 3 & 3 \\
\hline & & M & $\mathrm{S}$ & M & $\mathrm{S}$ & & M & $\mathrm{S}$ & M & $\mathrm{S}$ & M & $\mathrm{S}$ \\
\hline 4 & - & - & - & $\mathrm{d}$ & $\mathrm{d}$ & - & - & - & - & - & - & - \\
\hline 7 & - & - & - & $\mathrm{d}$ & $\mathrm{d}$ & - & $\mathrm{d}$ & d & $\mathrm{d}$ & d & d & d \\
\hline 11 & - & - & - & - & - & - & - & - & d & d & - & - \\
\hline 13 & - & - & - & - & - & - & - & - & d & - & - & - \\
\hline 23 & - & - & - & - & - & - & - & - & $\mathrm{d}$ & d & $\mathrm{d}$ & d \\
\hline 28 & - & - & d & $\mathrm{d}$ & $\mathrm{d}$ & - & - & - & - & - & - & $\mathrm{d}$ \\
\hline 40 & - & - & + & + & + & - & $\mathrm{d}$ & d & $\mathrm{d}$ & d & $\mathrm{d}$ & d \\
\hline
\end{tabular}

+ : positif (gélose violette); - : négatif (gélose jaune) ; d douteux (gélose présentant des zones jaune et violette); $\mathrm{M}$ : matin ; S : soir. Remarque : le concentré minéral était distribué pour la première fois lors de la traite du jour 0 au matin.

+: positive result (purple agar); -: negative result (yellow agar); d: questionable (yellow and purple agar); M: morning; $\mathrm{S}$ : evening. Complemented feed was distributed first time at milking of 0 day in the morning.

\subsubsection{Test à la pénicillinase et identification au Charm HVS-8100}

Le Delvotest $\mathrm{SP}^{\circledR}$ effectué en présence de pénicillinase n'a pas occasionné une diminution du pouvoir inhibiteur. L'agent inhibiteur n'appartient donc pas au groupe des antibiotiques à noyau $\beta$-lactame sensible à la pénicillinase.

Les échantillons de lait de la vache $n^{\circ} 40$ réagissant positivement ont été soumis à des essais d'identification de groupes d'antibiotiques par le Charm HVS-8100. Aucun des cinq groupes d'antibiotiques suivants : macrolides, aminoglycosides, sulfamides, tétracyclines et antibiotiques à noyau $\beta$-lactame n'a été mis en évidence.

\subsection{Essai 3}

Le troisième essai a donné uniformément des résultats négatifs au Delvotest $\mathrm{SP}^{\circledR}$.

\section{DISCUSSION ET CONCLUSIONS}

Les trois séries d'essai ont confirmé l'existence de résultats positifs avec Delvotest $\mathrm{SP}^{\circledR}$ dans le lait cru de certaines exploitations alors qu'un traitement médicamenteux ne pouvait être incriminé. Le régime alimentaire exerce une influence sur le résultat du test. Cette influence n'est pas encore expliquée mais la présence de compléments minéraux en alimentation hivernale peut être liée à une réponse positive.

La nature de ces inhibiteurs n'a pas encore été définie mais leur appartenance à un groupe d'antibiotiques tel que macrolides, sulfamides, aminoglycosides, tétracyclines ou antibiotiques à noyau $\beta$-lactame, n'a pas pu être confirmée.

Le complément minéral n'est pas le facteur directement responsable des réponses positives au Delvotest $\mathrm{SP}^{\circledR}$ mais apparaît comme un facteur déclenchant ou ampli- 
fiant un phénomène existant. Les essais effectués montrent qu'un second facteur dépendant du type de ration alimentaire influence le test : les réponses négatives obtenues lors du pâturage indiquent que la ration d'hiver (principalement la fraction ensilage) joue un rôle important. On n'a pas observé de Delvotest $\mathrm{SP}^{\circledR}$ faux positif en été avec du lait de vaches s'alimentant en pâtures et recevant un complément minéral identique à celui donné en hiver, période durant laquelle des réponses positives ont été observées.

Le facteur alimentaire pourrait être étudié en faisant varier la nature et la qualité de la ration alimentaire brute, ensilage de maïs, ensilage d'herbe, foin, préfané... D'autres explications peuvent aussi être recherchées du côté de l'équilibre ionique du lait et du $\mathrm{pH}$ perturbé par les variations du régime ali- mentaire. Avant tout, il serait opportun d'identifier le facteur inhibiteur.

\section{BIBLIOGRAPHIE}

[1] Mäyrä-Makinen A., Technological significance of residues for the dairy industry, in: Residues of Antimicrobial Drugs and Other Inhibitors in Milk, IDF Publication 9505, Bruxelles, 1995, pp. 136-143.

[2] Règlement (CEE) $\mathrm{n}^{\circ} 2377 / 90 \mathrm{du}$ Conseil du 26 juin 1990 établissant une procédure communautaire pour la fixation des limites maximales de résidus de médicaments vétérinaires dans les aliments d'origine animale, JO L224/1 du 18 juin 1990 (modifications ultérieures de ce règlement).

[3] Législation belge : Arrêté ministériel du 14 octobre 1994, Moniteur Belge du 2 décembre 1994, p 29910.

[4] Carlsson A., Björck L. The effect of some indigenous antibacterial factors in milk on the growth of Bacillus stearothermophilus var. Calidolactis, Milchwissenschaft, 42 (1987) 282-285. 